\section{Use of Astronomy Filters in Light Microscopy and Photomicrography}

\author{
Jörg Piper \\ Clinic Meduna, Bad Bertrich Germany \\ webmaster@prof-piper.com
}

\section{Introduction}

In addition to other determining factors, the optical quality of images is strongly dependent on the quality of the lenses and the spectral characteristics of the incident light in light microscopy as well as in astronomy. Chromatic aberration is one of the most important reasons for limitations of the hotgraphic quality. Ideal lenses that are completely free from such aberrations do not exist. A little chromatic aberration remains in "high-end" apochromatic lenses. Moreover, several lenses for special applications, e.g. microscopic objectives for long focal distances and $\frac{e}{n}$ thick glass plates $\mathbf{s}$ or objectives with an integrated iris $\mathbf{y}$ diaphragm for observations in dark field, are mostly not apochromatically corrected because of their optical compromises.

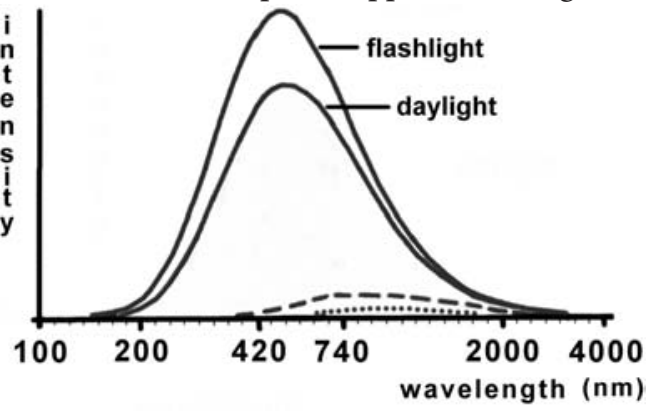

Fig. 1: Spectra and intensities of various lightsources (modified from Puchner, 2006). Dotted line: incandescent bulb $\left(2600^{\circ} \mathrm{K}\right)$, Broken line: halogen bulb $\left(3400^{\circ} \mathrm{K}\right)$, Full line: day light $\left(5600^{\circ} \mathrm{K}\right)$ and flash light $\left(5900^{\circ} \mathrm{K}\right)$

Optical image quality is also influenced by the light source and its spectrum. Conventional light bulbs, halogen lamps, mercury vapor or xenon arc lamps, daylight, and flashlight have different intensities and spectras (fig. 1). In a similar manner, celestial bodies that are observed by astronomers differ in their spectral chracteristics. Table 1 gives an overview of the characteristic wavelengths in the visible and non-visible light. Non-visible spectral components (infrared / IR and ultraviolet / UV radiation) may cause additional diminutions of image quality in microscopy and astronomy. They can lead to interferences with visible light-lower contour sharpness and color fringes can result from this. Especially, if digital cameras are used, the sharpness of the photo and the character and intensity of its colors can be compromised by unwanted IR and UV radiation. In astronomy, further negative effects can result from "light pollution", i.e. scattered terrestrial radiation (5). The higher the magnification of an optical system the higher the negative influences of these quality limiting factors.

A great many filters have been developed for astronomy applications in order to improve the optical quality of telescopes (refractors as well as reflector telescopes). These filters are constructed as interference filters that select and/or reject different spectral ranges within the visible and not-visible light spectra. They can block atmospheric stray light (skyglow and street light emissions), cut IR and UV light, or work as selective bandpass-filters. This way, spectral ranges that are relevant for the respective astronomical observation can be selected; fundamental improvements of astronomical images can result $(3,4,6,9,10)$. In this article I discuss some astronomical filters, suitable for microscopic use, which have been rigorously and successfully tested in practice.

\section{Materials and Methods}

Three types of astronomical interference-filters were evaluated, made by the Baader Planetarium Company, Germany: spectral cutters, bandpass-filters, and monochromatic filters. The filters were either inserted into the illuminating light path or mounted in front of the instrument's viewing heads or eye pieces. Observations were made in bright and dark field, phase and interference contrast, and polarized light conditions. Digital images were taken by a 7.1 Mp
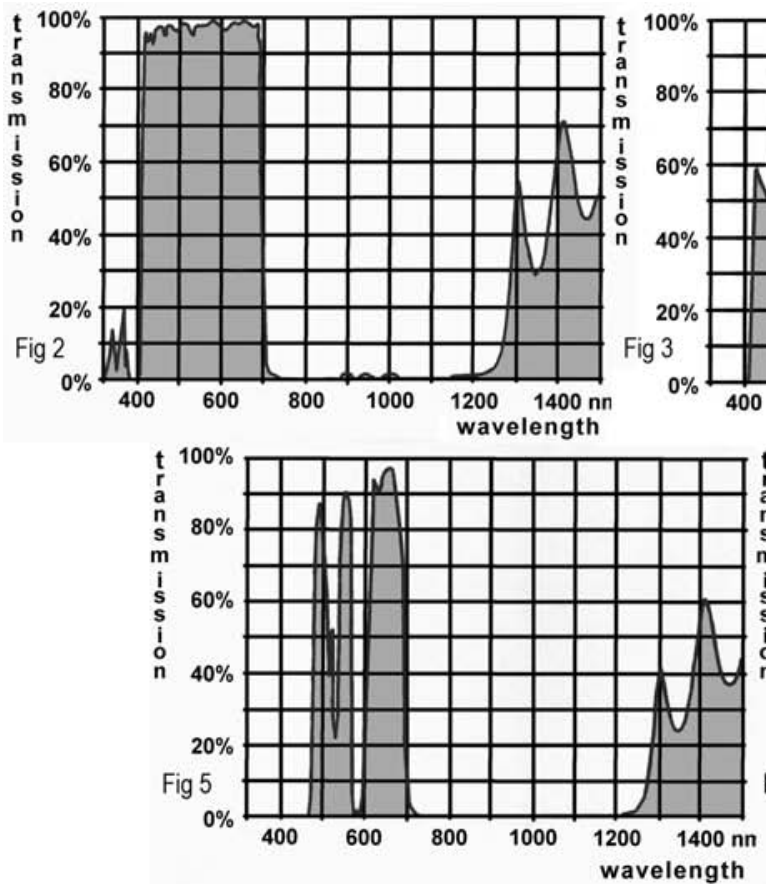
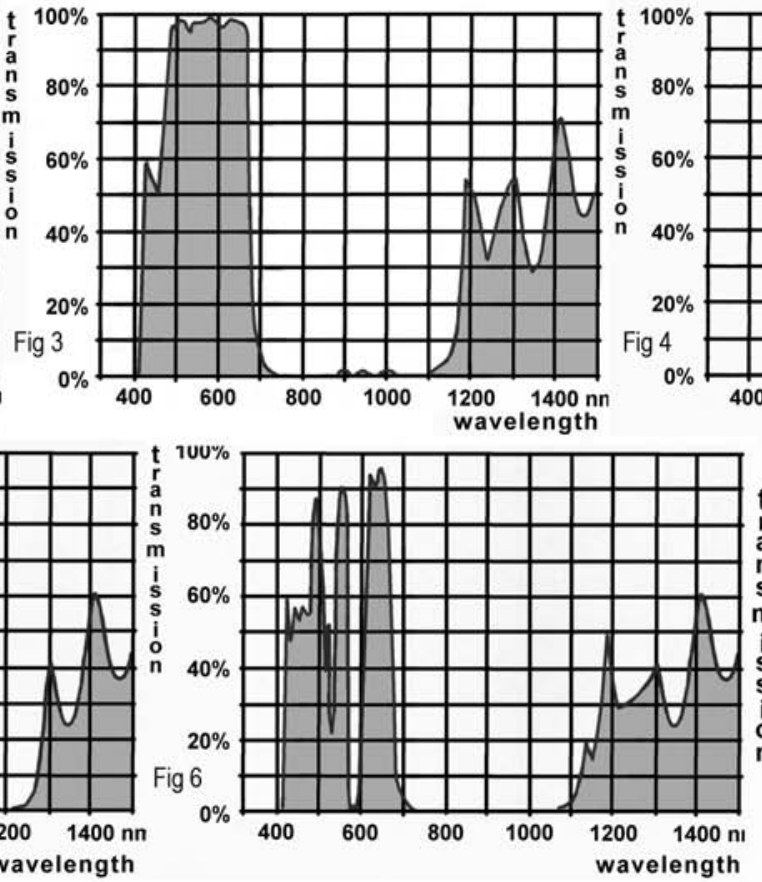
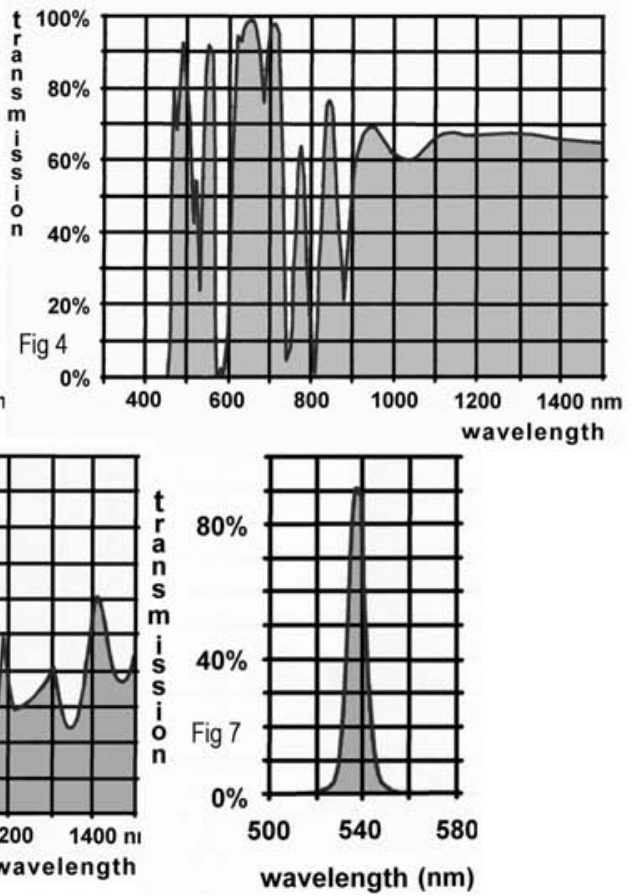

Fig. 2: Transmission of the Baader UV/IR-cut-filter (modified from Baader). Fig. 3: Transmission of the Baader fringe-killer-filter (modified from Baader). Fig. 4: Transmission of the Baader contrast-booster-filter (modified from Baader). Fig. 5: Transmission of the Baader contrast-booster-filter, combined with the UV/IR-cut-filter (modified from Baader). Fig. 6: Transmission of the Baader semi-apo-filter (modified from Baader). Fig. 7: Transmission of the Baader solar-continuum-filter (modified from Baader). 


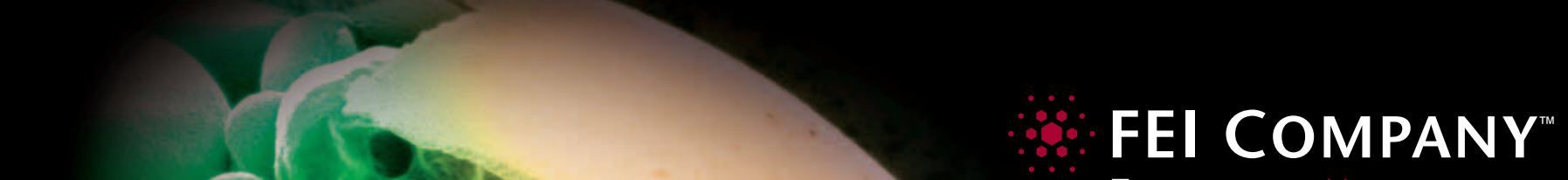

\section{Push the limits of ultra-high-resolution characterization, sample preparation and nanoprototyping}

The Helios NanoLab ${ }^{\mathrm{TM}}$, FEl's next generation DualBeam ${ }^{\mathrm{TM}}$, is in a class of its own when it comes to imaging, analysis and control of matter on the nanometer level. It produces the thinnest samples of the highest quality in a quick and easy way. It excels in preparing samples for almost any SEM/FIB application, with minimal damage to the sample. It pushes the limits in 2D and 3D nano characterization to deliver the most advanced integrated solutions for nanoprototyping. Its Titan $^{\mathrm{TM}}$-derived platform features unsurpassed deep nanometer resolution SEM performance to provide imaging and contrast of the highest quality with the best stability at the highest speed. All in all, Helios NanoLab is the perfect tool for pioneers who want to expand the boundaries in nanotechnologies.

For more information, www.fei.com 
Table 1: Ranges of wavelengths and typical nominal wavelengths of the spectral colors, (Achenlohe 2006, Puchner 2006, Sommers et al. 2006)

\begin{tabular}{|c|c|c|}
\hline Color & $\begin{array}{c}\text { Range of Wavelengths } \\
{[\mathbf{n m}]}\end{array}$ & $\begin{array}{c}\text { Nominal Wavelength } \\
{[\mathbf{n m}]}\end{array}$ \\
\hline ultraviolet (UV) & $<380$ & 400 \\
\hline violet & $380-420$ & 440 \\
\hline blue & $420-490$ & 465 \\
\hline magenta & $450-480$ & 510 \\
\hline cyan & $490-520$ & 540,546 \\
\hline green & $490-575$ & 580 \\
\hline yellow & $575-585$ & 590 \\
\hline orange & $585-650$ & 700 \\
\hline red & $650-750(780)$ & \\
\hline infrared (IR) & $>750(780)$ & \\
\hline
\end{tabular}

digital consumer camera (Olympus Camedia C 7070) with bulb and flash light. The microscope was equipped with plano apochromatic objectives and achromatic lenses, including some lenses specially adapted for long focal distances and dark field illumination.

The following filters were tested:

\section{UV/IR-cut-filter:}

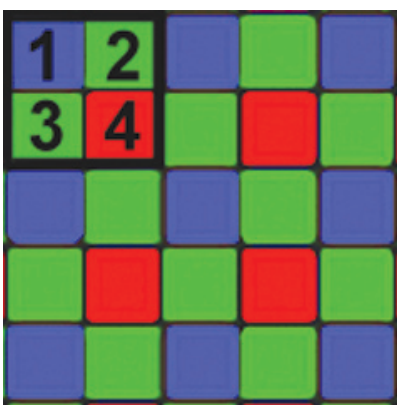

Fig. 8: $R G B$ pixel quartets according to the Bayer model.

\section{Contrast-booster-filter:}

This filter works as an RGB-intensifier, charcterized by $98 \%$ transmission for the spectral ranges of the threee primary colors red, green and blue. Transmissions are lower for cyan and yellow; violet is blocked (fig. 4). When combined with the UV/IR-cut-filter, the transmission is additionally reduced (range: $480-700 \mathrm{~nm}$ ), yielding a high selectivity for RGB (fig. 5).
Table 2: Characteristics of evaluated astronomical filters(modified from Baader, 2006)

\begin{tabular}{|c|c|}
\hline filter & maximum transmissions \\
\hline UV-IR-cut (UV-IR) & short-wave blue - medium-wave red $(410-690 \mathrm{~nm})$ \\
\hline fringe-killer & long-wave blue - short-wave red $(480-680 \mathrm{~nm})$ \\
\hline contrast-booster $(\mathrm{CB})$ & medium-wave blue - short-wave green $(460-510 \mathrm{~nm})$ \\
\hline & medium-wave green $(540-570 \mathrm{~nm})$ \\
\hline $\mathrm{CB}+$ UV-IR & orange - red $(600-730 \mathrm{~nm})$ \\
\hline & long-wave blue - short-wave green $(480-510 \mathrm{~nm})$ \\
\hline & long-wave green - yellow $(560-580 \mathrm{~nm})$ \\
\hline semi-apo & orange - short-wave red $(610-690 \mathrm{~nm})$ \\
\hline & short-wave blue - short-wave green $(420-510 \mathrm{~nm})$ \\
\hline & medium-wave green $(540-570 \mathrm{~nm})$ \\
\hline solar-continuum & orange - short-wave red $(610-680 \mathrm{~nm})$ \\
\hline
\end{tabular}

\section{Semi-APO-filter:}

The transmission of this filter is similar to the contrast-booster, so that this filter can also be regarded as an RGB-intensifier; blue light with shorter wavelength (>420 nm) can pass (fig. 6).

\section{Solar-continuum-filter:}

This filter for solar observations is constructed as a monochromatic green filter, maximum transmission: $538 \mathrm{~nm}$, half-intensity width: $10 \mathrm{~nm}$ (fig. 7).

\section{Results:}

\section{General aspects:}

All of the filters evaluated are available in $1-1 / 4$ " and 2 " sizes, so they can be combined with the usual microscopic apparatus. They are plane optically polished, high precision multicoated, and designed with non contacting mountings in order to achieve an equal and constant tension on each side of the glass. Thus, resolution, sharpness, and image homogenity are not detoriated and any image degradation is avoided when these filters are used. When spectral cutters and bandpass-filters are shifted into the illuminating light beam or mounted in front of viewing heads or eye pieces, all visible effects are equal, although different components of the light path are filtered in both modes of use. Therefore, these filters should preferably be integrated into the illuminating light. According to common use, the monochromatic filter was shifted into the illuminating light path as well.

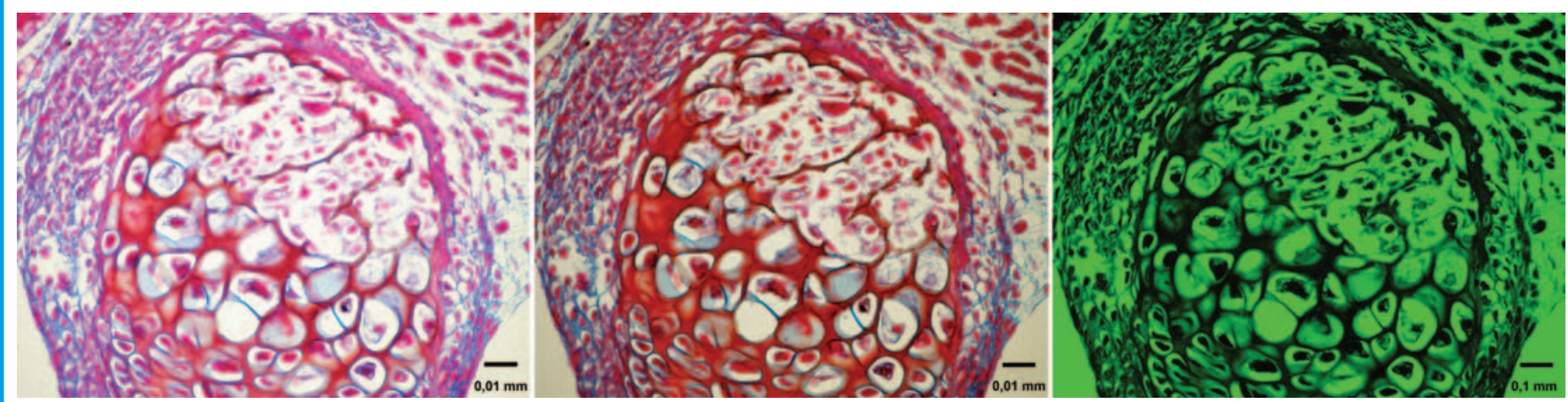

Fig. 9: Embryonic cartilage, bright field, objective 40x, eye piece 10x, flash, automatic TTL flashlight exposure metering, shutter speed: 1/2000 s. left: no filter, center: Baader contrast-booster-filter (RGB-intensifier), right: Baader solar-continuum-filter (monochromatic greenlight, 540nm). Editor's Note: Unfortunately, all RGB images suffered upon conversion to CMYK color space for printing. The difference between the left and center images was much more impressive than what we see here. 


\section{ENVIGION A NEW WAY OF COMBINED}

COMPETENCIES

\section{ZEISG NVision 40}

The new 3D CrossBeam ${ }^{\oplus}$ Workstation

that combines FIB and GEMINI ${ }^{\oplus}$ SEM columns

to a unique system enabling a variety of

outstanding nanotech capabilities
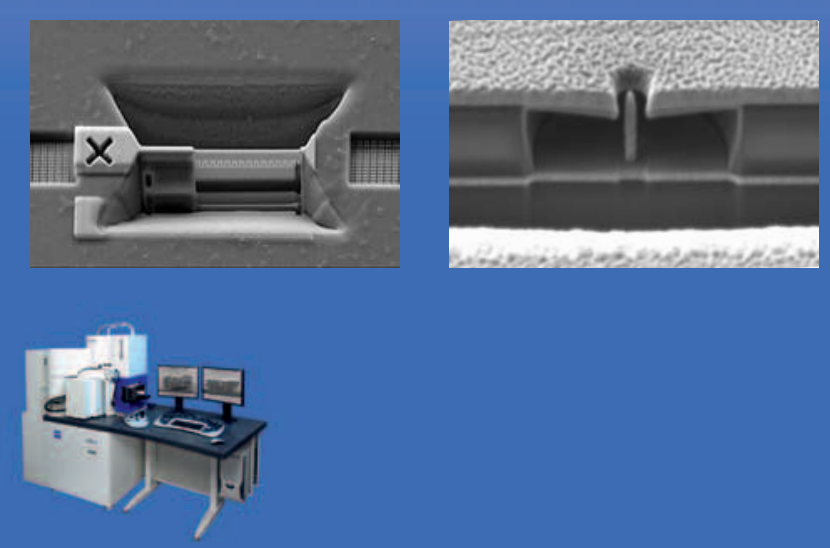

Enabling the Nano-Age World ${ }^{\bullet}$ NVision 40 - First-Class Efficiency and Convenience - Envision the Value of Precision

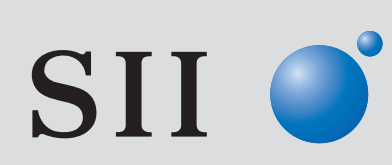

Carl Zeiss SMT Inc.

Tel. +1 978/826 1500 • Fax +1 978/5322503

info-usa@smt.zeiss.com•www.smt.zeiss.com/nts

SII NanoTechnology Inc.

www.siint.com

Tel. $+81 / 362800066$ 


\section{Special aspects:}

UV/IR-cut-filter:

This filter can be used as a heat protective filter, because it stays cool and will not shatter even if subjected to great thermal stress. As UV and IR radiations are completely blocked, the filter is also suitable for eye protection, when light sources with very intense radiation are used. As the complete range of visible light can pass ( $98 \%$ transmission), brightness and the visible colors of the specimens and background are not influenced. Potential im-

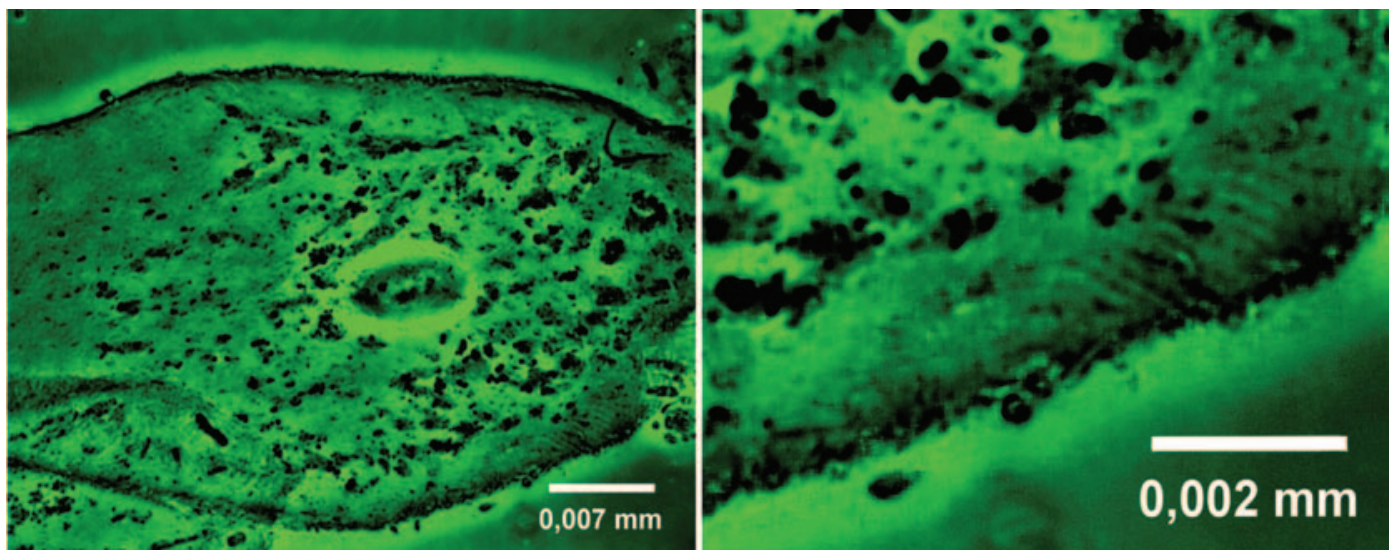

Fig. 10: Human epithelial cell from the oral mucosa, live-preparation, phase contrast, objective Oil 100x, eye piece 12,5 x, Baader solar-continuum filter, extraordinary improvements of sharpness and resolving power Left: Overview, Right: Detail from fig. 10a, subcellular structures with villous textures of the cell membrane age degradations caused by UV or IR light are reduced. When the microscope lenses are well coated and constructed as achromates or apochromates and when the digital camera is equipped with good UV- and IR-absorbers, this filter does not lead to further visible improvements of the respective images.

\section{Fringe-killer-filter:}

Blue or red fringing and bluish tint, caused by chromatic aberration, can be minimized, as IR, UV, violet, and parts of the blue light are blocked. Using this filter, sharpness and color balance can be improved, if poorly corrected optical systems are used. When high quality objectives and eyepieces are available, made with achromatic or apochromatic lenses, no additional improvments can be achieved in microscopic practice. Microscopic images appear with a slight greenish tint, as violet and blue components are subtracted. This effect may be beneficial in monochrome photomicrography.

\section{Contrast-booster:}

Color saturation, color contrast, and color purity of the resulting color separation are fundamentally improved in most microscopic applications, because the primary colors (red, green, blue) are enhanced selectively. Existing color errors can be eliminated and lack of contrast caused by bluish "hue" can be removed, especially when a flash is used for photomicrography. The precision of automatic TTL flash exposure metering can be improved when the contrast-booster-filter is used. Moreover, improvements of contour sharpness and light-dark contrast are achieveable, so that thin and low contrast structures appear with enhanced clarity. These effects can be intensified in some cases when this filter is used in combination with an UV/IR-cut-filter. See figure 9 left and center.

\section{Semi-Apo-filter:}

The transmission of this filter is similar to the Contrastbooster-filter, combined with an UV/IR-cut-filter. Contrary to the combination of these two filters, components of blue light with short wavelengths can pass. Therefore, the semi-apo-filter is free from any greenish tint. Microscopic images are characterized by saturated and warm colors, as typical for highly corrected apochromatic lenses. All fundamental optical improvements, described with regard to the contrast-booster-filter, are also achieveable with the help of the semi-apo-filter. There are small differences in the individual color tones. The RGB-enhancement can be intensified when two semiapo-filters are combined with each other. When a flash is used for photomicrography, the semi-apo-filter can be combined with the contrast-booster-filter. The saturation and clarity of colors can be additionally intensified in comparison with the contrast-boosterfilter used by itself or together with a UV/IR-cut-filter.

\section{Solar-continuum-filter:}

This filter works as a high quality monochromatic green filter, characterized by a small half-intensity width. When monochromatic light is used for illumination, any unsharpness based on chromatic aberration is eliminated; sharpness and contrast are maximized. The sensitivity of the human eye is optimized for green light, so that green images show maximum detail and tonal values. Moreover, the sharpness and resolving power of digital photomicrographs can be maximized by monochromatic green light, especially, when monochrome (e.g. red or blue) specimens are photographed. The usual color CCD sensors in cameras consist of a single layer of photodiodes or pixels. Each pixel is coated with a filter for one of the primary colors, red, green and blue (RGB). Each point in a color image is formed from a quartet of pixels: two pixels are covered with green filters, and the two neighboring pixels with red and blue filters (Bayer model, fig. 8). Thus, $50 \%$ of all pixels are sensitive only to green light, $25 \%$ to red and $25 \%$ to blue. When red or blue specimens are photographered in white light, only $25 \%$ of the pixels contribute to the digital image; the information in the other $75 \%$ is interpolated electronically by the camera. Loss of resolution and contour sharpness result from this. When monochromatic green light is used for photographic recording from red or blue specimens, $50 \%$ of all the pixels contribute to the digital image instead of $25 \%$. This way, the sharpness and resolution of the resulting digital photographs is improved. According to recommendations of the manufacturer, this filter should be combined with an UV/IR-cut-filter for eye protection, for it passes very intense light.

\section{Conclusions for practice:}

Selective bandpass-filters, working as RGB-intensifiers, can lead to extraordinary improvements of the image quality in microscopic observations as well as in photomicrography. Although all specimens appear in their natural colors, the augmentation of quality is comparable with the improving effects of dark or monochromatic green filters.

Both bandpass-filters, evaluated (contrast-booster- and semiapo-filter) are available at rather low prices. Nevertheless, the microscopic images, resulting from them, are characterized by a salutary apochromatic "flair". Even when apochromates are available, these 
filters can lead to additional visible improvements of the respective color resolution. Therefore, these filters may be regarded as very useful tools well suited for microscopic equipment.

Monochromatic green filters, available for solar observations, can also be used in microscopic fields very well. They offer special adventages, when sharpness and resolving power of monochrome images are to be maximized and digital cameras are utilized for photomicrography equipped with the usual RGB CCD-chips. For this application, a monochromatic green filter can lead to an optimized sharpness and resolution when combined with apochromatic lenses. The price of the solar-continuum-filter is equal to a bandpass-filter.

Spectral cutters, constructed as UV- and IR-blockers or fringekillers, do not lead to visible improvements of microscopic images, when achromatic or apochromatic lenses are available. The quality of digital microscopic photographs are usually not improved by these filters, if the digital camera is equipped with effective internal UV- and IR-absorbers. On the other hand, improvements in image quality may be seen for low-end, inexpensive, optical apparatus. These filters are suitable for heat- and eye protection if very intense light sources are used. Some improving effects that can be achieved with the help of astro-filters are demonstrated in figs. 9 and 10.

A suitable quartett of astro-filters might consist of the following components: Contrast-booster-filter, Semi-apo-filter, Solar-continuum-filter and UV/IR-cut-filter. These four filters can be purchased for about $\$ 200$ or $\$ 250$ US. Their optical benefit is comparable with improvements achieveable by apochromatic lenses constructed for high end systems for high end prices. The resulting quality of apochromates can be improved furthermore, when these filters are used. Thus, I would like to suggest these filters for all microscopists who are interested in optimizing their work.

\section{References:}

(1) Achenlohe, A.: Color and light, colorimetry (in German), 2006, http://www. actoid.com/webdesign/farblichtsehen/Farbmetrik.htm

(2) Baader, T.: Baader-Planetarium, color filters (descriptions in German and English), 2006. http://www.baader-planetarium.de/zubehoer/okularseitiges_ zubeh/farbfilter.htm

(3) Brown, B.: Light pollution filters - a spectral examination of the most popular light pollution filters in astronomy, 2006. http://home.comcast. net/ mcculloch-brown/astro/LPRFilters.html

(4) Dobbins, A.: What shall we do with „refraktor-blues“? (in German) Astronomie Heute, 9, 2004. http://www.baader-planetarium.de/suw_artikel/download/filtertest_ahoi.pdf

(5) Kornreich, D.: How does light pollution affect astronomers? Astronomy department at Cornell University, 2006. http://curious.astro.cornell.edu/question. php?number $=194$

(6) Marling, J.B.: Light pollution reduction by nebular filter, 2006. http://home. earthlink.net/ astro-app/horsehead/Marling.htm.

(7) Puchner, R.: Light and colors, light regarded as electromagnetic wave (in German), 2006. http://www.puchner.org/Fotografie/technik/physik/licht.htm

(8) Sommers, H.-J., Motzko, M., Oberhage, R.: Light and colors (in German), 2006. http://www.theo-phys.uni-essen.de/tp/onlinehelp/Colors/index.html

(9) Varden, T.: Filters, 2006. http://www.astro-tom.com/technical_data/filters. htm

(10) Weisheit, B.: Perfection for a 100 Euros surcharge- Color correction filters for Fraunhofer-refractor-telescopes in parallel tests (in German). Sterne und Weltraum, 8/03, 78-83, 2003. http://www.baader-planetarium.de/suw_artikel/ download/contrast_booster.pdf

\section{STOP HASSLING WITH MULTIPLE SERVICE CONTRACTS!}

START by putting all of our instruments under one service contract with MAS (regardless of make or model). Our expert EM SERVICE GROUP has the knowledge and skills to keep your instrument working at its best.

\section{TEM'S / SEM'S PREP EQUIPMENT SPECIAL SERVICES}

HITACHI TOPCON JOEL ISI

\section{AMRAY CAMBRIDGE}

\section{VACUUM COATERS \\ PLASMA ASHERS SPUTTER COATERS \\ ION MILLS}

STAGES

BEAM BLANKERS
CUSTOM DEVICES

\section{NEW NEW NEW}

\section{Authorized service representatives}

for Gatan preparation equipment

and Topcom TEM'S.
Contracts and On-Demand Emergency Service at

Reasonable Rates from Factory Trained Specialist

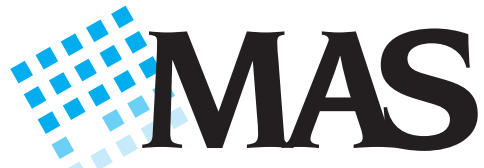

3945 Lakefield Court Suwanee, Georgia 30024 770-866-3200 FAX 770-866-3259 ADVANGED ANALYTIGAL PRODUGTS AND SERVIGES 